\title{
Efficacy of Integrative Chinese-Western Medicine Treatments for COVID-19 Patients in ICU in Yichang, Hubei, China
}

\section{Ziwen Yuan ( $\nabla$ yuanziwen@xjtufh.edu.cn )}

Xi'an Jiaotong University Medical College First Affiliated Hospital https://orcid.org/0000-0001-5647$289 X$

\section{Min Zhang}

Fujian University of Traditional Chinese Medicine

Jingcheng Li

900th Hospital of PLA

\section{Ting Peng}

Fujian University of Traditional Chinese Medicine

\section{Chen Liang}

Fujian University of Traditional Chinese Medicine

Zhengfeng Lin

Fujian University of Traditional Chinese Medicine

\section{Quan Ming}

Third People's Hospital of Yichang

\section{Qiuping Peng}

Third People's Hospital of Yichang

\section{Run Fang}

Third People's Hospital of Yichang

\section{Weijun Zhao}

Yichang Hospital of Traditional Chinese Medicine

\section{Bin Luo}

Fujian University of Traditional Chinese Medicine Hongbo Liu

Fujian University of Traditional Chinese Medicine

\section{Xiaojie Wei}

Fujian University of Traditional Chinese Medicine

\section{Wusong Huang}

Fujian University of Traditional Chinese Medicine

\section{Yanfeng Shao}

Fujian University of Traditional Chinese Medicine 


\section{Shengnan Xiong}

Fujian University of Traditional Chinese Medicine

\section{Research}

Keywords: COVID-19, ICU, critical care, Integrative Chinese-Western Medicine, Mortality

Posted Date: May 15th, 2020

DOI: https://doi.org/10.21203/rs.3.rs-27000/v1

License: (c) (1) This work is licensed under a Creative Commons Attribution 4.0 International License. Read Full License 


\section{Abstract}

Background The prognosis of severe COVID-19 patients is poor. There are currently no definitely effective vaccines or antivirus drugs for COVID-19.

Methods This prospective cohort study compared the efficacy and safety of integrative Chinese-Western medicine (ICWM) treatments with Western medicine (WM) treatments in severe or critically ill patients. The outcomes included: mortality, hospital stay in ICU, days with ventilator-assisted ventilation, etc.

Results A total of 36 confirmed COVID-19 patients in ICU were included. The median age of patients was 66 years (IQR: 53-77.5), and there were 16 female patients (44.4\%). There were no significant differences in laboratory tests and complications after treatments between groups. A total of 18 (50\%) patients died during hospitalization, and the mortality in the ICWM group (28.6\%) was significantly lower than that of the WM group $(63.6 \%$, adjusted $P=0.031)$. And the time of assisted ventilation was shorter in the ICWM group (adjusted $P=0.67$ ). However, the median hospital stay was significantly longer in the ICWM group (18 vs. 14 days, adjusted $P \otimes 0.05$ ).

Conclusions ICWM treatments could significantly reduce the mortality and improve the clinical symptoms for severe or critically ill patients with COVID-19, and it was safe and cost-effective to add Chinese medicine.

\section{Background}

COVID-19 has been rapidly spreading around the world [1]. As of April 16, 2020, there were a total of 83,799 cases of confirmed COVID-19 and 3,352 deaths across China, and the number reached 2006,042 and 136,119 in countries and regions outside of China respectively, which seriously endangered the health of people around the world and affected the social life and economy [2]. Most of the infected patients have mild clinical manifestations and good prognosis [3]. And multiple organ failure and acute respiratory distress syndrome (ARDS) often occur in severe patients, who are often accompanied by chronic diseases, and the prognosis is poor [4].

At present, there are no definitely effective vaccines or pharmacological treatments for COVID-19 [5]. The disease also belongs to the category of Traditional Chinese Medicine (TCM) epidemic, the cause is the body felt by the epidemic, and the location is in the lung according to TCM theory. Based on the previous experience of TCM in the treatment of SARS and COVID-19, the sixth version of the program on the diagnosis and treatment of COVID-19 in China (February 18, 2020) introduced the TCM recommendations for COVID-19 [6]. So we employed integrative Chinese-Western Medicine (ICWM) treatments for some patients admitted to the intensive care unit (ICU) with confirmed infection with 2019-nCoV since February 20,2020 . The study intends to analyze the clinical characteristics of severe and critically ill patients and to explore the efficacy of ICWM treatments compared with Western medicine treatments alone.

\section{Methods}


This study was a prospective cohort study that observed patients admitted to ICU with confirmed COVID19 in the Third People's Hospital of Yichang, Hubei, China (a designated infectious diseases hospital). We defined patients who received only Western medicine treatments as the Western medicine (WM) group, and those who have added TCM decoction to Western medicine treatment were classified as the ICWM group. The differences will be observed in mortality, days with assisted ventilation, hospital stay, and improvement in clinical symptoms between the two groups of patients. The study has been reviewed by the Ethics Review Committee of the Third People's Hospital Affiliated to Fujian University of Chinese Medicine, and all patients have signed informed consent.

\section{Populations}

All enrolled patients were severe or critically ill with COVID-19. The diagnostic and classification criteria were referred to that of the interim guideline on COVID-19 of the World Health Organization (WHO) and China [6,7]. Detailed inclusion criteria are as follows: 1 . Age $\geq 18$ years; 2 . Positive 2019-nCoV PCR test of respiratory specimens before admission; 3 . Severe pneumonia with/without the following situations: ARDS, multiple organ failure, sepsis, or shock. Exclusion criteria: 1. Those who took chloroquine phosphate; 2. Those who have been enrolled in trials of other TCM drugs.

\section{Western medicine treatments}

The following antiviral drugs were used according to the patient conditions: a-interferon, ribavirin, abidol or lopinavir, and ritonavir tablets [6]. In principle, no more than three antiviral drugs were used at the same time. Antibiotics were given to patients with fever and significantly elevated peripheral blood leukocytes or positive blood bacterial culture. All patients were given oxygen inhalation and monitored for vital signs. Ventilator-assisted ventilation was considered for patients with respiratory distress and/or hypoxemia when oxygen inhalation cannot relieve them. Corresponding supportive treatments would be given if the patient has complications or adverse events associated with the disease itself or treatments.

\section{TCM treatments}

TCM syndrome differentiation and formulation referred to the diagnosis and treatment program on the new coronavirus infectious pneumonia (trial sixth edition) in China (See details in suppl. Table 1) [6]. The program divided severe or critically ill patients with COVID-19 into three manifestations: severe (1. pestilence toxin retention in lung, 2. intense heat in qi and ying systems), and critically (3. internal obstruction and external collapse). Recommended formulations of corresponding clinical stages and subtypes are summarized in supplement Table 1.

\section{Outcome measurement}

All resident doctors were responsible for recording the patient's epidemiology, clinical manifestations, laboratory tests, chest CT, treatment plan, and clinical outcomes in the electronic medical records, and the trained personnel recorded the above information in a special data collection form. The data collection form was reviewed daily, and errors and omissions found were queried and corrected timely. 
The primary outcome was mortality; other outcomes included: mortality, hospital stay in ICU, days with ventilator-assisted ventilation, the degree of dyspnea, and complications during hospitalization.

Ventilator-assisted ventilation used only for rescue before death is not recorded. We used a visual analog scale (VAS) [8] to evaluate the degree of dyspnea, with that a score of 0 indicates no dyspnea, and 10 indicates unbearable dyspnea. The degree of dyspnea was marked on a scale of about $10 \mathrm{~cm}$ by the patient. VAS and laboratory tests were assessed at admission and $10 \pm 2$ days after admission. Neutrophil-lymphocyte-ratio (NLR) was calculated by blood neutrophil count /lymphocyte count. Complications recorded included acute cardiac injury (ACI), acute kidney injury (AKI), acute liver injury (ALI), acute respiratory distress syndrome (ARDS)\and arrhythmia.

\section{Statistical analysis}

Measurement data were expressed by median (IQR), and count data were expressed by rate or composition ratio. The mean comparison between the two groups was performed by t-test or Kruskal Wallis rank-sum test; rate comparison was performed by chi-square test or Fisher exact test. Risk factors at admission related to TCM treatments and disease outcomes were screened based on univariate analysis results and previous literature. Multivariate regression analysis was used to compare the effects of the two treatments after adjusting for age, gender, and above risk factors. Spearman correlation analysis was used to compare the correlation between blood oxygen saturation and VAS scores for dyspnea. Then we took subgroup analysis by patients of different ages ( $</ \geq 60$ years) and gender. Take $a=0.05$ (two sides). Empower (R) (www.empowerstats.com; $X \& Y$ solutions, Inc., Boston MA) and R software, version 3.1 .2 (http://www.r-project.org) were used for all statistical analyses.

\section{Results}

\section{Patients and Baseline Characteristics}

A total of 36 confirmed COVID-19 patients in ICU were included. And all patients had a 2019nCoV-related epidemiological history. The WM group finally involved 22 patients and 14 patients for the ICWM group. The median age of patients was 66 years (IQR: 53-77.5), and there were 16 female patients (44.4\%). Among the patients, the most common comorbidities were hypertension $(50.0 \%)$ and diabetes $(30.6 \%)$. There were $28(77.8 \%)$ patients with fever and cough. Most patients have moderate dyspnea with a VAS score of 6 (IQR: 5-7), while the median oxygen saturation $\left(\mathrm{SaO}_{2}\right)$ under oxygen inhalation was $94 \%$ (IQR: 91\%-96.2\%). The median time from onset to admission in the ICWM group was 8 days (IQR: 3.2-20), and 5 days (IQR: $3-7)$ in the WM group ( $P=0.152)$. There was no significant difference in other baseline characteristics between the ICWM group and the WM group except for mean arterial pressure (Table 1).

\section{Laboratory tests and complications}

There was no significant difference in laboratory tests between the two groups at admission and 10 days after treatments (Table 2). The lymphocyte, neutrophil count, and NLR of the ICWM group were slightly lower than those of the WM group at admission, but the difference was not significant; after 10 days of 
treatment, the lymphocyte count of two groups was equal and the neutrophil count and NLR in the ICWM group were slightly lower. The mean C-reactive protein (CRP) was similar at admission (WM: $58.9 \mathrm{mg} / \mathrm{L}$, ICWM: $49.2 \mathrm{mg} / \mathrm{L})$; while it seemed lower in the ICWM group $(73.2 \mathrm{mg} / \mathrm{L})$ than that of WM group (33.2 $\mathrm{mg} / \mathrm{L}, P=0.094)$ at about 10 days after admission. And there were no significant differences in liver function, kidney function, blood coagulation function, and blood gas analysis after treatment.

ARDS occurred in 29 (80.6\%) patients (WM: 81.8\%, ICWM: 78.6\%), and $13(36.1 \%)$ patients were given non-invasive ventilator-assisted ventilation (WM: 45.5\%, ICWM: 21.4\%), and 14 (38.9\%) patients received invasive ventilator-assisted ventilation (WM: $31.8 \%$, ICWM: 50.0\%) within 72 hours of admission. Other more common complications during hospitalization were shock (total 47.2\%, WM 54.5\%, ICWM 35.7\%), ALI (total 33.3\%, WM 27.3\%, ICWM 42.9\%) and ACI (total 25.0\%, WM 22.7\%, ICWM 28.6 \%), etc. However, there was no significant difference between the two groups (Table 3 ).

\section{Clinical outcome}

A total of $18(50 \%)$ patients died during hospitalization, and the mortality in the ICWM group (28.6\%) was significantly lower than that of the WM group (63.6\%). After using a multivariate regression analysis to adjust the related risk factors, there was still significant difference between the two groups $(P=0.031)$ (Table 4). Besides, after 10 days of treatment, the median VAS score of the ICWM group was 4, while the score of the WM group was 7 (adjusted $P \otimes 0.05$ ). And the time of assisted ventilation was shorter in ICWM group comparing with WM group, but not significant (adjusted $P=0.67$ ). However, the median hospital stay was significantly longer in the ICWM group (18 vs. 14 days, adjusted $P \otimes 0.05$ ); and we found the same result after excluding dead patients ( 20 vs. 15.5 days, adjusted $P=0.563$ ). In the subgroup analysis, we found similar trend in the above outcomes for the young /aged and male /female patients (suppl. Table2).

\section{Discussion}

2019-nCoV is a new coronavirus that is not completely equivalent to MERS and SARS [9], and humans are universally susceptible to it.[10] but elderly patients of are in more serious condition [11, 12]. The median age of patients in our ICU was 66 years old, which was similar to other report $[3,13,14]$. And our patients were severe or critically ill; in the first 72 hours of admission, 75\% patients were given mechanical ventilation because of refractory respiratory distress or hypoxemia. Recently, Mahase [15] indicated that most patients require mechanical ventilation within 24 hours of critical care. Studies have also found that severe cases tend to have lower lymphocytes counts, higher leukocytes counts, NLR and inflammatory parameters, such as CRP $[16,17]$. Among the above indicators reflecting the severity of COVID-19, there was no significant difference at admission between the two groups in our study.

The utilization of TCM for COVID-19 is substantial in China, over $85 \%$ of confirmed cases included TCM treatments nationally (Wuhan over 67\%) [18]. Back in SARS epidemic, a report released by WHO and a meta-analysis indicated that ICWM treatment had the potential benefits to alleviate symptoms, reduce complications and cost, and was safe [19, 20]. Recently, Luo et. al [21] found that TCM had an advantage 
in keeping microenvironmental balance in treating COVID-19. Another study investigating the efficacy of ICWM treatment on COVID-19 found that ICWM treatments could improve clinical symptoms better and faster [22]. But TCM treatments in the study included TCM decoction, Chinese patent medicine and TCM injection simultaneously [22].

This study was a prospective cohort study of COVID-19 patients in ICU. It analyzed the clinical characteristics of 36 severe or critically patients, and the efficacy and safety of ICWM treatments. The total mortality was $50 \%$ which was similar to the current report, and the mortality in the ICWM group was lower than previously reported.[13] We found that adding TCM decoction to WM treatments could significantly reduce the mortality of COVID-19. After adjusting the risk factors associated to severity at admission, including VAS, NLR and CRP, there was still a significant difference between the two groups. And the degree of dyspnea was also alleviated more in the ICWM group (lower VAS score). However, we found that the hospital stay of patients in the ICWM group was longer. It seemed to be contradictory to the current report [22]. One explanation was that our patients were all severe or critically ill. Firstly, we assumed that the same severity will bring the same mortality. Secondly, our results presented the similar severity at admission between groups but a lower level of inflammation during hospitalization in the ICWM group. Then we suspected that TCM smoothed the rising slope of the inflammatory storm of these patients who should have died, but also bring longer inflammatory response time. As discovered by a network pharmacology study, Qing-fei -pai-du decoction can regulate excessive immune response by Th17 cell differentiation, T cell and B cell signaling, and TNF signaling pathway in COVID-19 [23]. We may call it "time for life".

In terms of safety, the added TCM treatment did not significantly affect the coagulation, kidney, cardiac and liver function and other aspects of COVID-19 patients, and did not increase the incidence of COVID19-related complications during hospitalization. We can see that the addition of TCM was safe for severe or critically ill COVID-19 patients. Until now, there are no antiviral drugs specifically targeting 2019-nCoV [24]. Remdesivir have been indicated promising anti-viral effects in both intracellular and extracellular [25, 26]; but its clinical efficacy and safety have not yet been proven [10]. Besides, the currently used antiviral drugs have different degrees of damage to the kidney, liver, bone marrow, and peripheral nerves [24]. And with the advantages of efficacy and safety and low cost (about 3-5 dollars per day), TCM treatments may be recommended currently, especially intensive care resources are scarce.

The main limit of this study was that it was observational, sample size was small, and there was an imbalance between the two groups at admission. However, we adjusted the risk factors in Multivariate regression analysis to minimize the effect of these baseline biases on the research results. Besides, due to the urgency of the epidemic situation and the research conditions, and the $\mathrm{SaO}_{2}$ was measured under oxygen inhalation, the patient's dyspnea was evaluated using the patient's self-assessed VAS score [8], which may lack objectivity, but can reflect the changes in the subjective feelings of patients to a certain extent. This study revealed that the addition of TCM decoction significantly reduced mortality and improved the clinical symptoms. But it is unknown how TCM plays a role in this disease, its pharmacological mechanism should be further explored. 


\section{Conclusions}

For severe or critically ill patients with COVID-19, ICWM treatments could significantly reduce the mortality and improve the clinical symptoms. The potential mechanism may be that TCM smoothed the rising slope of the inflammatory storm. And it was safe and cost-effective to add TCM decoction to Western medicine treatments.

\section{Abbreviations}

WM: Western medicine, ICWM: integrative Chinese-Western medicine, ICU: intensive care unit, TCM: Traditional Chinese Medicine, ACl: acute cardiac injury, AKI: acute kidney injury, ALI: acute liver injury, ARDS: acute respiratory distress syndrome, COPD: chronic obstructive pulmonary disease, CKD: chronic kidney disease, MAP: Mean arterial pressure, VAS: visual analog scale. $\mathrm{SaO}_{2}$ : oxygen saturation, WBC: white blood cell, NLR: neutrophil-lymphocyte-ratio, PT: prothrombin time, APTT: activated partial thromboplastin time, BUN: blood urea nitrogen, SCr: serum creatinine, CK: creatinekinase, LDH: lactate dehydrogenase, ALT: alanine aminotransferase, AST: aspartate aminotransferase, CRP: C-reactive protein.

\section{Declarations}

\section{Acknowledgments}

Not applicable.

\section{Authors' contributions}

Ziwen Yuan and Min Zhang drafted the main manuscript and made statistical analysis. Ting Peng, Chen Liang, Zhengfeng Lin, Qiuping Peng, Hongbo Liu, Xiaojie Wei, Wusong Huang, Run Fang, Yanfeng Shao and Shengnan Xiong collected and sorted data. Bing Luo and Weijun Zhao drafted the text about traditional Chinese medicine treatment. Jingcheng $\mathrm{Li}$ and Quan Ming revised the manuscript for important intellectual contribution. All authors read the final manuscript and approved submissions.

\section{Funding}

None.

\section{Availability of data and materials}


Extra data is available by email to Dr. Min Zhang (Minzhang-2018@163.com) on a reasonable request.

\section{Ethics approval and consent to participate}

The study has been reviewed by the Ethics Review Committee of the Third People's Hospital Affiliated to Fujian University of Chinese Medicine, and all patients have signed informed consent.

\section{Consent for publication}

Not applicable.

\section{Competing interests}

The authors declare that they have no competing interests.

\section{References}

1. Zhu N, Zhang D, Wang W, Li X, Yang B, Song J, et al. A Novel Coronavirus from Patients with Pneumonia in China, 2019. N Engl J Med. 2020;382:727-33. doi:10.1056/NEJMoa2001017.

2. Bogoch II, Watts A, Thomas-Bachli A, Huber C, Kraemer M, Khan K. Potential for global spread of a novel coronavirus from China. JOURNAL OF TRAVEL MEDICINE. 2020;27. doi:10.1093/jtm/taaa011.

3. Wang D, Hu B, Hu C, Zhu F, Liu X, Zhang J, et al. Clinical Characteristics of 138 Hospitalized Patients With 2019 Novel Coronavirus-Infected Pneumonia in Wuhan, China. JAMA. 2020. doi:10.1001/jama.2020.1585.

4. Chen N, Zhou M, Dong X, Qu J, Gong F, Han Y, et al. Epidemiological and clinical characteristics of 99 cases of 2019 novel coronavirus pneumonia in Wuhan, China: a descriptive study. LANCET. 2020;395:507-13. doi:10.1016/S0140-6736(20)30211-7.

5. Shanmugaraj B, Siriwattananon K, Wangkanont K, Phoolcharoen W. Perspectives on monoclonal antibody therapy as potential therapeutic intervention for Coronavirus disease-19 (COVID-19). Asian Pac J Allergy Immunol. 2020;38:10-8. doi:10.12932/AP-200220-0773.

6. National Health Commission and National Administration of Traditional Chinese Medicine. The diagnosis and treatment program on the new coronavirus infectious pneumonia (trial version 6). National Health Commission and National Administration of Traditional Chinese Medicine. 2020.

7. World Health Organization. Clinical management of severe acute respiratory infection (SARI) when COVID-19 disease is suspected: Interim guidance. World Health Organization. 2020. 
8. Szentes BL, Schwarzkopf L, Kirsch F, Schramm A, Leidl R. Measuring quality of life in COPD patients: comparing disease-specific supplements to the EQ-5D-5L. Expert Rev Pharmacoecon Outcomes Res. 2019:1-7. doi:10.1080/14737167.2019.1662302.

9. Prompetchara E, Ketloy C, Palaga T. Immune responses in COVID-19 and potential vaccines: Lessons learned from SARS and MERS epidemic. Asian Pac J Allergy Immunol. 2020;38:1-9. doi:10.12932/AP-200220-0772.

10. Adhikari SP, Meng S, Wu YJ, Mao YP, Ye RX, Wang QZ, et al. Epidemiology, causes, clinical manifestation and diagnosis, prevention and control of coronavirus disease (COVID-19) during the early outbreak period: a scoping review. Infectious Diseases of Poverty. 2020;9:29. doi:10.1186/s40249-020-00646-x.

11. Lian J, Jin X, Hao S, Cai H, Zhang S, Zheng L, et al. Analysis of Epidemiological and Clinical features in older patients with Corona Virus Disease 2019 (COVID-19) out of Wuhan. CLINICAL INFECTIOUS DISEASES. 2020. doi:10.1093/cid/ciaa242.

12. Mo $P$, Xing $Y$, Xiao $Y$, Deng $L$, Zhao Q, Wang $H$, et al. Clinical characteristics of refractory COVID-19 pneumonia in Wuhan, China. CLINICAL INFECTIOUS DISEASES. 2020. doi: 10.1093/cid/ciaa270.

13. Bhatraju PK, Ghassemieh BJ, Nichols M, Kim R, Jerome KR, Nalla AK, et al. Covid-19 in Critically III Patients in the Seattle Region - Case Series. N Engl J Med. 2020. doi:10.1056/NEJMoa2004500.

14. Liu K, Chen Y, Lin R, Han K. Clinical features of COVID-19 in elderly patients: A comparison with young and middle-aged patients. J Infect. 2020. doi:10.1016/j.jinf.2020.03.005.

15. Mahase E. Covid-19: most patients require mechanical ventilation in first 24 hours of critical care. BMJ. 2020;368:m1201. doi:10.1136/bmj.m1201.

16. Qin C, Zhou L, Hu Z, Zhang S, Yang S, Tao Y, et al. Dysregulation of immune response in patients with COVID-19 in Wuhan, China. CLINICAL INFECTIOUS DISEASES. 2020. doi:10.1093/cid/ciaa248.

17. Zhang W, Zhao Y, Zhang F, Wang Q, Li T, Liu Z, et al. The use of anti-inflammatory drugs in the treatment of people with severe coronavirus disease 2019 (COVID-19): The Perspectives of clinical immunologists from China. CLINICAL IMMUNOLOGY. 2020;214:108393. doi:10.1016/j.clim.2020.108393.

18. Chan KW, Wong VT, Tang S. COVID-19: An Update on the Epidemiological, Clinical, Preventive and Therapeutic Evidence and Guidelines of Integrative Chinese-Western Medicine for the Management of 2019 Novel Coronavirus Disease. Am J Chin Med. 2020:1-26. doi:10.1142/S0192415X20500378.

19. World Health Organization. Clinical trials on treatment using a combination of Traditional Chinese medicine and Western medicine. World Health Organization. 2004;pp:1-194.

20. Liu X, Zhang M, He L, Li Y. Chinese herbs combined with Western medicine for severe acute respiratory syndrome (SARS). Cochrane Database Syst Rev. 2012;10:D4882. doi:10.1002/14651858.CD004882.pub3.

21. Luo E, Zhang D, Luo H, Liu B, Zhao K, Zhao Y, et al. Treatment efficacy analysis of traditional Chinese medicine for novel coronavirus pneumonia (COVID-19): an empirical study from Wuhan, Hubei Province, China. Chin Med. 2020;15:34. doi:10.1186/s13020-020-00317-x. 
22. Xia W, An C, Zheng C, Zhang J, Huang M, Wang Y, et al. Clinical study on 34 cases of new coronavirus pneumonia treated with integrated traditional Chinese and Western medicine. Journal of Traditional Chinese Medicine. 2020.

23. Zhao J, Tian S, Yang J, Liu J, Zhang W. Investigating the mechanism of Qing-Fei-Pai-Du-Tang for the treatment of novel coronavirus pneumonia by network pharmacology. Chinese Traditional Herbal Drugs. 2020:829-35.

24. Lodise TP, Rybak MJ. COVID-19: Important Therapy Considerations and Approaches in this Hour of Need. PHARMACOTHERAPY. 2020. doi:10.1002/phar.2396.

25. Sheahan TP, Sims AC, Zhou S, Graham RL, Pruijssers AJ, Agostini ML, et al. An orally bioavailable broad-spectrum antiviral inhibits SARS-CoV-2 in human airway epithelial cell cultures and multiple coronaviruses in mice. Sci Transl Med. 2020. doi:10.1126/scitransImed.abb5883.

26. Yang Y, Islam MS, Wang J, Li Y, Chen X. Traditional Chinese Medicine in the Treatment of Patients Infected with 2019-New Coronavirus (SARS-CoV-2): A Review and Perspective. International Journal of Biological Sciences. 2020;16:1708-17. doi:10.7150/ijbs.45538.

\section{Tables}

Table 1. Baseline Characteristics of COVID-19 Patients in ICU. 


\begin{tabular}{|c|c|c|c|c|}
\hline & \multicolumn{3}{|c|}{ Median (Q1-Q3) /N (\%) } & \multirow[t]{2}{*}{$P$-value } \\
\hline & Total & $\mathrm{WM}$ & ICWM & \\
\hline $\mathrm{N}$ & 36 & 22 & 14 & \\
\hline Age (years) & $66(53-77.5)$ & $66(52.2-76)$ & $68(55-77)$ & 0.721 \\
\hline Sex & & & & 0.734 \\
\hline male & $20(55.6 \%)$ & $13(59.1 \%)$ & $7(50.0 \%)$ & \\
\hline female & $16(44.4 \%)$ & $9(40.9 \%)$ & $7(50.0 \%)$ & \\
\hline \multicolumn{5}{|l|}{ Comorbidities } \\
\hline Hypertension & $18(50.0 \%)$ & $10(45.5 \%)$ & $8(57.1 \%)$ & 0.733 \\
\hline Cardiovascular disease & $9(25.0 \%)$ & $4(18.2 \%)$ & $5(35.7 \%)$ & 0.267 \\
\hline Diabetes & $11(30.6 \%)$ & $8(36.4 \%)$ & $3(21.4 \%)$ & 0.467 \\
\hline Malignancy & $2(5.6 \%)$ & $0(0.0 \%)$ & $2(14.3 \%)$ & 0.144 \\
\hline Cerebrovascular disease & $7(19.4 \%)$ & $4(18.2 \%)$ & $3(21.4 \%)$ & 0.81 \\
\hline COPD & $5(13.9 \%)$ & $2(9.1 \%)$ & $3(21.4 \%)$ & 0.357 \\
\hline $\mathrm{CKD}$ & $6(16.7 \%)$ & $4(18.2 \%)$ & $2(14.3 \%)$ & 0.76 \\
\hline HIV infection & $0(0 \%)$ & $0(0 \%)$ & $0(0.0 \%)$ & - \\
\hline \multicolumn{5}{|l|}{ Signs and symptoms } \\
\hline Fever & $28(77.8 \%)$ & $16(72.7 \%)$ & $12(85.7 \%)$ & 0.441 \\
\hline Cough & $28(77.8 \%)$ & $16(72.7 \%)$ & $12(85.7 \%)$ & 0.441 \\
\hline Expectoration & $20(55.6 \%)$ & $13(59.1 \%)$ & $7(50.0 \%)$ & 0.734 \\
\hline Fatigue & $19(52.8 \%)$ & $12(54.5 \%)$ & $7(50.0 \%)$ & 0.79 \\
\hline Pharyngalgia & $7(19.4 \%)$ & $4(18.2 \%)$ & $3(21.4 \%)$ & 0.334 \\
\hline Diarrhea & $2(5.6 \%)$ & $2(9.1 \%)$ & $0(0.0 \%)$ & 0.511 \\
\hline Nausea & $2(5.6 \%)$ & $0(0.0 \%)$ & $2(14.3 \%)$ & 0.144 \\
\hline Dizziness & $4(11.1 \%)$ & $1(4.5 \%)$ & $3(21.4 \%)$ & 0.277 \\
\hline Headache & $3(8.3 \%)$ & $1(4.5 \%)$ & $2(14.3 \%)$ & 0.547 \\
\hline Anxiety & $2(5.6 \%)$ & $1(4.5 \%)$ & $1(7.1 \%)$ & 0.74 \\
\hline Insomnia & $13(36.1 \%)$ & $7(31.8 \%)$ & $6(42.9 \%)$ & 0.723 \\
\hline Temperature $\left({ }^{\circ} \mathrm{C}\right)$ & $36.8(36.5-37.8$ & $36.9(36.5-37.7)$ & 36.7 (36.5-37.9) & 0.424 \\
\hline $\mathrm{SaO} 2(\%)$ & $94.0(91.0-96.2$ & $94.0(91.2-96.8)$ & $94.5(91.0-96.0)$ & 0.935 \\
\hline Heart rate (beats/min) & $88.5(81.5-97.0$ & $89.0(82.5-95.5)$ & $87.0(75.2-97.8)$ & 0.548 \\
\hline MAP (mmHg) & $93.3(88.3-99.7$ & $90.3(86.8-95.8)$ & $99.0(93.8-105.2)$ & 0.008 \\
\hline
\end{tabular}




\begin{tabular}{|c|c|c|c|c|}
\hline VAS for dyspnea & $6.0(5.0-7.0)$ & $5.5(5.0-7.0)$ & $6.0(5.0-6.8)$ & 0.583 \\
\hline Time from onset to admission (d) & $5.5(3.0-10.0)$ & $5.0(3.0-7.0)$ & $8.0(3.2-20.0)$ & 0.152 \\
\hline
\end{tabular}

Abbreviations: WM: Western medicine, ICWM: integrative Chinese-Western medicine, COPD: chronic obstructive pulmonary disease, CKD: chronic kidney disease, SaO2: oxygen saturation, MAP: Mean arterial pressure, VAS: visual analog scale.

Table 2. Changing of Laboratory Tests of COVID-19 Patients.

\begin{tabular}{|c|c|c|c|c|c|c|c|}
\hline & \multicolumn{2}{|c|}{ at admission } & \multirow[t]{2}{*}{$P$-value } & \multicolumn{2}{|c|}{10 days after admission } & \multirow{2}{*}{$P$-value } & \multirow{2}{*}{$\begin{array}{l}\text { Abbreviations: } \\
\text { Western medicine, ICWM }\end{array}$} \\
\hline & WM & ICWM & & $\mathrm{WM}$ & ICWM & & \\
\hline $\mathrm{N}$ & 22 & 14 & & 22 & 14 & & integrative \\
\hline WBC $\left(\times 10^{9} / \mathrm{L}\right)$ & $4.8(4.0-7.8)$ & $5.6(4.3-6.9)$ & 0.972 & $11.9(9.6-13.6)$ & $9.2(6.2-17.6)$ & 0.575 & Western medicine, WBC: \\
\hline Neutrophil $\left(\times 10^{9} / \mathrm{L}\right)$ & $3.7(2.1-6.7)$ & $3.9(3.2-5.8)$ & 0.923 & $10.9(7.6-12.4)$ & $7.0(4.7-13.0)$ & 0.31 & blood \\
\hline Lymphocyte $\left(\times 10^{9} / \mathrm{L}\right)$ & $0.7(0.6-1.0)$ & $0.8(0.5-1.0)$ & 0.503 & $0.6(0.4-0.9)$ & $0.6(0.3-0.8)$ & 0.675 & neutrophil- \\
\hline NLR & $5.1(2.6-9.3)$ & $4.2(3.2-10.3)$ & 0.246 & $17.4(12.1-23.7)$ & $12.5(6.4-43.5)$ & 0.889 & lymphocyte-ratio, \\
\hline Hemoglobin $(\mathrm{g} / \mathrm{L})$ & $109.0(104.0-122.0)$ & $114.5(91.5-122.2)$ & 0.934 & $109.0(94.2-121.2)$ & $100.0(83.2-103.8)$ & 0.214 & prothrombin time, \\
\hline PT (s) & $11.2(10.6-11.4)$ & $10.5(10.0-11.8)$ & 0.359 & $11.8(11.1-12.4)$ & $11.3(10.9-11.6)$ & 0.514 & activated \\
\hline APTT (s) & $30.0(24.7-34.9)$ & $28.6(24.0-29.3)$ & 0.606 & $24.0(22.5-30.1)$ & $28.9(25.2-32.9)$ & 0.103 & thromboplastin time, BUN: \\
\hline D-dimer (mg/L) & $0.9(0.6-2.8)$ & $1.0(0.6-6.6)$ & 0.84 & $5.4(2.1-15.4)$ & $9.2(4.0-12.9)$ & 0.715 & blood urea nitrogen, SCr: \\
\hline BUN (mmol/L) & $5.1(4.4-11.3)$ & $6.0(4.4-7.9)$ & 0.41 & $8.6(6.1-13.3)$ & $5.9(4.5-9.1)$ & 0.087 & 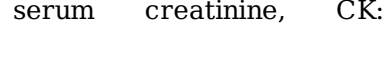 \\
\hline $\mathrm{SCr}(\mu \mathrm{mol} / \mathrm{L})$ & 65.7 (63.3-89.7) & $61.2(50.3-69.2)$ & 0.177 & $74.3(51.0-93.2)$ & $61.7(45.9-79.6)$ & 0.274 & (1) \\
\hline $\mathrm{CK}(\mathrm{U} / \mathrm{L})$ & 99.5 (63.2-149.8) & $59.5(35.2-81.8)$ & 0.183 & $146.0(43.0-181.0)$ & $56.0(24.0-156.0)$ & 0.202 & renyarogenase, \\
\hline LDH (U/L) & $413.0(226.0-477.0)$ & $308.0(255.0-420.0)$ & 0.247 & $532.5(384.5-704.5)$ & $382.0(271.0-549.0)$ & 0.335 & \\
\hline ALT (U/L) & $26.0(18.0-35.0)$ & $16.0(10.2-35.5)$ & 0.177 & $36.0(21.5-71.0)$ & $29.0(18.8-53.0)$ & 0.771 & \\
\hline AST (U/L) & $30.0(21.0-48.0)$ & $25.0(22.2-31.0)$ & 0.112 & $28.0(22.0-43.5)$ & $24.0(17.5-50.0)$ & 0.827 & \\
\hline $\mathrm{PH}$ & $7.4(7.4-7.5)$ & $7.4(7.4-7.4)$ & 0.832 & $7.4(7.3-7.5)$ & $7.4(7.4-7.5)$ & 0.316 & \\
\hline Lactate $(\mathrm{mmol} / \mathrm{L})$ & $2.0(1.5-2.4)$ & $2.0(1.9-2.5)$ & 0.742 & $1.9(1.4-3.0)$ & $1.9(1.4-2.4)$ & 0.812 & \\
\hline CRP & $61.0(23.6-89.6)$ & $27.4(15.4-65.0)$ & 0.525 & $30.7(8.6-115.1)$ & $28.0(10.0-42.5)$ & 0.328 & \\
\hline
\end{tabular}


Table 3. Comparisons of Complications During Hospitalization between WM and ICWM Group.

\begin{tabular}{lcccc}
\hline & \multicolumn{3}{c}{$\mathrm{N}(\%)$} & \multirow{2}{*}{$P$-value } \\
\cline { 2 - 4 } & Total & WM & ICWM & \\
\cline { 2 - 4 } $\mathrm{N}$ & 36 & 22 & 14 & 0.712 \\
Acute cardiac injury & $9(25.0 \%)$ & $5(22.7 \%)$ & $4(28.6 \%)$ & 0.547 \\
Arrhythmia & $3(8.3 \%)$ & $1(4.5 \%)$ & $2(14.3 \%)$ & 0.217 \\
Acute kidney injury & $8(22.2 \%)$ & $3(13.6 \%)$ & $5(35.7 \%)$ & 0.471 \\
Acute liver injury & $12(33.3 \%)$ & $6(27.3 \%)$ & $6(42.9 \%)$ & 0.322 \\
Shock & $17(47.2 \%)$ & $12(54.5 \%)$ & $5(35.7 \%)$ & 0.810 \\
\hline
\end{tabular}

Abbreviations: WM: Western medicine, ICWM: integrative Chinese-Western medicine, ARDS: acute respiratory distress syndrome.

Table 4. Comparisons of Clinical Outcomes Between WM and ICWM group. 


\begin{tabular}{|c|c|c|c|c|}
\hline & \multirow{2}{*}{$\begin{array}{c}\text { Outcomes } \\
\text { N (\%) /Median (Q1-Q3) }\end{array}$} & \multicolumn{3}{|c|}{$\beta$ (95\%CI)/OR(95\%CI) $P$-value } \\
\hline & & Model 1 & Model 2 & Model 3 \\
\hline \multicolumn{5}{|l|}{ Death } \\
\hline WM & $14(63.6 \%)$ & Ref & Ref & Ref \\
\hline ICWM & $4(28.6 \%)$ & $0.23(0.05,0.97) 0.046$ & $0.20(0.04,0.91) 0.037$ & $0.00(0.00,0.57) 0.031$ \\
\hline \multicolumn{5}{|c|}{ Time of assisted ventilation (d) } \\
\hline $\mathrm{WM}$ & $11(1.5-15)$ & Ref & Ref & Ref \\
\hline ICWM & $7(1.2-11)$ & $-1.03(-6.29,4.22) 0.703$ & $-0.49(-5.73,4.74) 0.854$ & $1.28(-4.54,7.11) 0.671$ \\
\hline \multicolumn{5}{|l|}{ VAS at 10 days } \\
\hline \multicolumn{5}{|l|}{ of admission } \\
\hline WM & $7(5-8)$ & Ref & Ref & Ref \\
\hline ICWM & $4(3-4.8)$ & $-2.41(-3.67,-1.15) 0.001$ & $-2.17(-3.43,-0.90) 0.002$ & $-2.65(-3.96,-1.34) 0.001$ \\
\hline \multicolumn{5}{|l|}{ Hospital stay (d) } \\
\hline WM & $14(6.2-20.2)$ & Ref & Ref & Ref \\
\hline ICWM & $18(12-29.8)$ & \multicolumn{3}{|c|}{$6.23(-0.36,12.83) 0.0736 .59(-0.19,13.37) 0.06616 .39(10.81,21.97)<0.001$} \\
\hline
\end{tabular}

Model 1: Non-adjusted; Model 2: adjust for: Age and Sex; Model 3: adjust for: Age; Sex; Hypertension; Diabetes; Malignancy; Cerebrovascular disease; Chronic kidney disease; Cardiovascular disease; COPD; VAS at admission; Mean arterial pressure(mmHg); CRP at admission; NLR at admission. Abbreviations: WM: Western medicine, ICWM: integrative Chinese-Western medicine, VAS: visual analog scale.

\section{Supplementary Files}

This is a list of supplementary files associated with this preprint. Click to download.

- Supplements.docx 